\title{
Adaptive Asymptotic Tracking of Spacecraft Attitude Motion with Inertia Matrix Identification
}

\author{
Jasim Ahmed, ${ }^{*}$ Vincent T. Coppola, ${ }^{\dagger}$ and Dennis S. Bernstein ${ }^{\ddagger}$ \\ University of Michigan, Ann Arbor, Michigan 48109-2140
}

\begin{abstract}
The problem of a spacecraft tracking a desired trajectory is defined and addressed using adaptive feedback control. The control law, which has the form of a sixth-order dynamic compensator, does not require knowledge of the inertia or center of mass of the spacecraft. A Lyapunov argument is used to show that tracking is achieved globally. A simple spin about the intermediate principal axis and a coning motion are commanded to illustrate the control algorithm. Finally, periodic commands are used to identify the inertia matrix of the spacecraft.
\end{abstract}

\section{Introduction}

$\mathbf{T}$ HE present generation of spacecraft require attitude control systems that provide rapid acquisition, tracking, and pointing capabilities, while the equations that govern large-angle maneuvers are coupled and nonlinear. As such, control system design must consider the nonlinear dynamics. Furthermore, because the mass properties of the spacecraft may be uncertain or may change due to fuel usage and articulation, it is necessary for the control system to be able to adapt to changes in mass distribution. In this paper, we present a feedback control algorithm that achieves large-angle tracking of velocity and attitude commands in spite of inertia uncertainty. Furthermore, we use this tracking algorithm to identify the spacecraft inertia matrix.

Although open-loop control strategies have been developed for large-angle maneuvers, ${ }^{1,2}$ these controllers are generally sensitive to spacecraft parameter uncertainties, unexpected disturbances, and initial attitude rates. In Refs. 3 and 4, globalreorientationis achieved using body-fixed actuators without knowledge of the inertia of the spacecraft. These algorithms are based on decreasing the energy of the spacecraft until the desired orientation is achieved.

The attitude tracking problem has been consideredin Ref. 5 using a locally convergentadaptive algorithm, whereas adaptive feedback linearization is used in Ref. 6 to achieve tracking. However, the method of Ref. 6 requires measurements of the orientation and angular velocity of the spacecraft as well as angular acceleration. In Ref. 7, p. 428, an adaptive tracking scheme was developed that is globally valid except for a singularity. A switching maneuver is used to avoid the singularity. In Ref. 8 the authors develop a tracking control law that depends on a parameter that is required to be sufficiently small. Bounds for this parameter depend on knowledge of the largest and smallest principal moments of inertia. An adaptive tracking control law developed in Ref. 9 is able to guarantee convergence to a set consisting of four states, one of which is the desired state.

In the present paper, we develop an adaptive tracking control law that requires no knowledge of the spacecraft inertia matrix. The control law is globally valid, that is, free of singularities, and has the form of a sixth-order proportional-integral compensator. The algorithm is adaptive in the sense of Ref. 10, Chapter 1, because it contains adjustable parameters and a mechanism for adjusting these parameters.

Received July 21, 1997; presented as Paper 97-3530 at the AIAA Guidance, Navigation, and Control Conference, New Orleans, LA, Aug. 11-13, 1997; revision received March 13, 1998; accepted for publication March 23, 1998. Copyright (C) 1998 by the American Institute of Aeronautics and Astronautics, Inc. All rights reserved.

*Student, Department of Aerospace Engineering. E-mail: jasim@engin. umich.edu. Student Member AIAA.

'Assistant Professor, Department of Aerospace Engineering. E-mail: coppola@engin.umich.edu. Member AIAA.

$\doteqdot$ Professor, Department of Aerospace Engineering. E-mail: dsbaero@ engin.umich.edu.
We also show that the tracking algorithm is able to identify the spacecraft inertia matrix by using periodic command signals. A related technique is given in Refs. 9 and 11 where richness conditions are required to guarantee inertia matrix identification. However, a method to determine whether a particular command signal satisfies these conditions is not given.

We illustrate the tracking algorithm by commanding the spacecraft to perform a constant spin about an axis fixed to the spacecraft with the axis required to point in a given inertial direction. We perform the simulations for the case in which this axis is coincident with the intermediateprincipalaxis. Although the algorithmrequires three torque inputs, we require no knowledge of the spacecraft inertia. The problem of stabilizing a spacecraft about an intermediate principal axis without inertial pointing has been studied in Refs. 12 and 13 using a single control torque. Next, a coning motion is commanded using the tracking algorithm. Finally, identification of the spacecraft inertia matrix is illustrated using a periodic command signal.

\section{Equations of Motion}

The spacecraft is modeled as a rigid body with actuators that provide body-fixed torques about three mutually perpendicular axes that define a body-fixed frame $\mathcal{B}$ located at a point $P$ on the spacecraft. The point $P$ need not be located at the center of mass of the spacecraft whose location may be unknown. For each axis we assume that a body-fixed torque can be produced by employing a pair of actuators to produce equal and opposite forces perpendicular to the line joining the actuators. These lines joining the actuators need not pass through $P$. We translate the body frame $\mathcal{B}$ to the center of mass of the spacecraft to produce another frame $\mathcal{B}_{\mathrm{cm}}$ with axes $X_{\mathcal{B}_{\mathrm{cm}}}, Y_{\mathcal{B}_{\mathrm{cm}}}$, and $Z_{\mathcal{B}_{\mathrm{cm}}}$ that is located at the center of mass of the spacecraft and that has the same orientation as frame $\mathcal{B}$. Thus the orientation of $\mathcal{B}_{\mathrm{cm}}$ is the same as the orientation of $\mathcal{B}$.

For $t \geq 0$, the equations of motion of the spacecraft are given by

$$
\begin{gathered}
J \dot{\Omega}=-\Omega^{\times} J \Omega+u \\
\dot{\epsilon}=\frac{1}{2}\left(\epsilon^{\times} \Omega+\zeta \Omega\right) \\
\dot{\zeta}=-\frac{1}{2} \epsilon^{T} \Omega
\end{gathered}
$$

where $\Omega=\Omega(t) \in \Re^{3}$ is the inertial angular velocity of the spacecraft with respect to an inertial frame $\mathcal{I}$,

$$
J=\left[\begin{array}{lll}
J_{11} & J_{12} & J_{13} \\
J_{12} & J_{22} & J_{23} \\
J_{13} & J_{23} & J_{33}
\end{array}\right]
$$

is the constant positive-definite inertia matrix of the spacecraft, both expressed in $\mathcal{B}_{\mathrm{cm}}, u=u(t) \in \mathfrak{R}^{3}$ is the vector of control torques, $(\epsilon, \zeta)=[\epsilon(t), \zeta(t)] \in \mathfrak{R}^{3} \times \mathfrak{R}$ are the Euler parameters ${ }^{14}$ representing the orientation of $\mathcal{B}_{\mathrm{cm}}$ with respect to an inertial frame $\mathcal{I}$ satisfying the constraint

$$
\epsilon^{T} \epsilon+\zeta^{2}=1
$$


Corrections to this paper:

In Equation 9 there should be a minus sign on the first term of the left hand side i.e . -Imu*lepsilon instead of $\backslash m u^{*}$ lepsilon

Similarly in equation 10, i.e -Imu* zeta instead of $\left|m u^{*}\right| z e t a$ 
and ( $\left.{ }^{\circ}\right)$ denotes the derivative with respect to time $t$. The notation $a^{\times}$for $a=\left[\begin{array}{lll}a_{1} & a_{2} & a_{3}\end{array}\right]^{T}$ denotes the skew-symmetric matrix

$$
a^{\times}=\left[\begin{array}{ccc}
0 & -a_{3} & a_{2} \\
a_{3} & 0 & -a_{1} \\
-a_{2} & a_{1} & 0
\end{array}\right]
$$

The rotation matrix $B=B(\epsilon, \zeta) \in \mathrm{SO}(3)$ relating $\mathcal{B}_{\mathrm{cm}}$ to $\mathcal{I}$ is given by

$$
B=\left(\zeta^{2}-\epsilon^{T} \epsilon\right) I_{3 \times 3}+2 \epsilon \epsilon^{T}-2 \zeta \epsilon^{\times}
$$

where $I_{3 \times 3}$ is the $3 \times 3$ identity matrix. We assume that the orientation and angular velocity of frame $\mathcal{B}$ (and hence of frame $\mathcal{B}_{\mathrm{cm}}$ ) with respect to the inertial frame $\mathcal{I}$ are known for all time $t \geq 0$. However, $J$ is assumed to be unknown. Note that no knowledge of the center of mass of the spacecraft is required.

Let the desired rotationalmotion of the spacecraftbe described by the attitude motion of a frame $\mathcal{D}$ whose orientation with respect to $\mathcal{I}$ is specified by the Euler parameters $(\xi, \mu)=[\xi(t), \mu(t)] \in \mathfrak{R}^{3} \times \mathfrak{R}$ satisfying

$$
\xi^{T} \xi+\mu^{2}=1
$$

Let $D=D(\xi, \mu) \in \mathrm{SO}(3)$ be the corresponding rotation matrix given by

$$
D=\left(\mu^{2}-\xi^{T} \xi\right) I_{3 \times 3}+2 \xi \xi^{T}-2 \mu \xi^{\times}
$$

and let $v=v(t) \in \mathfrak{R}^{3}$ denote the angular velocity of $\mathcal{D}$ with respect to $\mathcal{I}$ and expressed in $\mathcal{D}$ by

$$
v=2(\mu \dot{\xi}-\dot{\mu} \xi)-2 \xi^{\times} \dot{\xi}
$$

Let the time derivative of $v$ be denoted by $\dot{v}=\dot{v}(t) \in \mathfrak{R}^{3}$. Then $\dot{v}$ is given by

$$
\dot{v}=2(\mu \ddot{\xi}-\ddot{\mu} \xi)-2 \xi \times \ddot{\xi}
$$

Let $(\varepsilon, \eta)=[\varepsilon(t), \eta(t)] \in \mathfrak{R}^{3} \times \mathfrak{R}$ be the Euler parameters representing the orientation of frame $\mathcal{B}_{\mathrm{cm}}$ with respect to $\mathcal{D}$. Then $(\varepsilon, \eta)$ satisfy

$$
\varepsilon^{T} \varepsilon+\eta^{2}=1
$$

The Euler parameters $(\varepsilon, \eta)$ are related to $(\xi, \mu)$ and $(\epsilon, \zeta)$ by the quaternion multiplication rule (Ref. 14, p. 17)

$$
\begin{gathered}
\varepsilon=\mu \epsilon-\zeta \xi+\epsilon^{\times} \xi \\
\eta=\mu \zeta+\xi^{T} \epsilon
\end{gathered}
$$

The corresponding rotation matrix $C=C(\varepsilon, \eta) \in \mathrm{SO}(3)$ is given by

$$
C=\left(\eta^{2}-\varepsilon^{T} \varepsilon\right) I_{3 \times 3}+2 \varepsilon \varepsilon^{T}-2 \eta \varepsilon^{\times}
$$

and is related to $B$ and $D$ by

$$
C=B D^{T}
$$

The angular velocity $\omega=\omega(t) \in \mathfrak{R}^{3}$ of $\mathcal{B}_{\mathrm{cm}}$ with respect to $\mathcal{D}$ and expressed in $\mathcal{B}_{\mathrm{cm}}$ is then

$$
\omega=\Omega-C v
$$

We assume that the desired maneuver is specified in terms of $(\xi, \mu)$, which are assumed to be $C^{2}$ functions, and that measurements of $\Omega$ and the attitude of frame $\mathcal{B}$ (and thus frame $\mathcal{B}_{\mathrm{cm}}$ ) with respect to $\mathcal{I}$ are available. Using Eqs. (5-7), the quantities $v, \dot{v}$, and $D$ can be computed from $(\xi, \mu)$. Furthermore, because $(\epsilon, \zeta)$ and $B$ can be calculated from the measurements of the attitude of $\mathcal{B}_{\mathrm{cm}}$, it follows that $\varepsilon, \eta$, and $C$ can be determined using Eqs. (9-11). The angular velocity $\omega$ is then determined using Eq. (13). The following problem expresses the requirement that the attitude and angular velocity of frames $\mathcal{B}_{\mathrm{cm}}$ and $\mathcal{D}$ should coincide asymptotically.
Tracking Problem. Let $\xi:[0, \infty) \rightarrow \Re^{3}$ and $\mu:[0, \infty) \rightarrow \Re$ be given $C^{2}$ Euler parameters satisfying Eq. (4) for all $t \geq 0$. Find a dynamic feedback control law of the form

$$
\begin{aligned}
\dot{\hat{\alpha}} & =f(\hat{\alpha}, \omega, \varepsilon, \eta, v, \dot{v}) \\
u & =g(\hat{\alpha}, \omega, \varepsilon, \eta, v, \dot{v})
\end{aligned}
$$

such that $C \rightarrow I_{3 \times 3}$ and $\omega \rightarrow 0$ as $t \rightarrow \infty$.

Note that the attitude convergence condition $C \rightarrow I_{3 \times 3}$ does not imply the angular velocity convergence condition $\omega \rightarrow 0$. This observation is simply a reflection of the mathematical fact that if a differentiable function converges to a constant, then the derivative of the function need not converge to zero. For example, consider $f(x)=\sin \left(x^{4}\right) /(x+1)$ for $x \rightarrow \infty$. Then $\lim _{x \rightarrow \infty} f(x)=0$, whereas $\lim _{x \rightarrow \infty} f^{\prime}(x)$ does not exist.

From Eqs. (8) and (11) it follows that $\varepsilon \rightarrow 0$ if and only if $C \rightarrow I_{3 \times 3}$. Hence the tracking problem is solved if and only if $\varepsilon \rightarrow 0$ and $\omega \rightarrow 0$. Rewriting Eqs. (1-3) in terms of $\omega, \varepsilon$, and $\eta$, we obtain the seven-dimensional system

$$
\begin{gathered}
J \dot{\omega}=-(\omega+C v)^{\times} J(\omega+C v)+J\left(\omega^{\times} C v-C \dot{v}\right)+u \\
\dot{\varepsilon}=\frac{1}{2}\left(\varepsilon^{\times} \omega+\eta \omega\right) \\
\dot{\eta}=-\frac{1}{2} \varepsilon^{T} \omega
\end{gathered}
$$

These equations describe the motion of the spacecraft with respect to $\mathcal{D}$. We observe that the tracking problem has been converted into an asymptotic stabilization problem for $\omega$ and $\varepsilon$ in Eqs. (16-18).

\section{Adaptive Control Law}

In this section, we present a feedback control law that asymptotically tracks a desired maneuver and thus satisfies the requirements of the tracking problem. The control law is global in the sense that asymptotic tracking is achieved for arbitrary initial conditions.

We observe that the inertia parameters $J_{i j}$, where $i, j=1,2,3$, appear linearly in Eq. (16). To isolate these parameters, we define a linear operator $L: \mathfrak{R}^{3} \rightarrow \mathfrak{R}^{3 \times 6}$ acting on $a=\left[\begin{array}{lll}a_{1} & a_{2} & a_{3}\end{array}\right]^{T}$ by

$$
L(a)=\left[\begin{array}{cccccc}
a_{1} & 0 & 0 & 0 & a_{3} & a_{2} \\
0 & a_{2} & 0 & a_{3} & 0 & a_{1} \\
0 & 0 & a_{3} & a_{2} & a_{1} & 0
\end{array}\right]
$$

Letting

$$
\alpha \triangleq\left[\begin{array}{llllll}
J_{11} & J_{22} & J_{33} & J_{23} & J_{13} & J_{12}
\end{array}\right]^{T}
$$

it follows that

$$
J a=L(a) \alpha
$$

Equation (16) can now be rewritten in the form

$$
J \dot{\omega}=F(\omega, C, v, \dot{v}) \alpha+u
$$

where $F: \mathfrak{R}^{3} \times \mathfrak{R}^{3 \times 3} \times \mathfrak{R}^{3} \times \mathfrak{R}^{3} \rightarrow \mathfrak{R}^{3 \times 6}$ is defined by

$$
F(\hat{\omega}, \hat{C}, \hat{v}, \hat{\rho}) \triangleq-(\hat{\omega}+\hat{C} \hat{v})^{\times} L(\hat{\omega}+\hat{C} \hat{v})+L\left(\hat{\omega}^{\times} \hat{C} \hat{v}-\hat{C} \hat{\rho}\right)
$$

Theorem 1. Assume that $v$ and $\dot{v}$ are bounded and let $K_{1} \in \mathfrak{R}^{3 \times 3}$, $K_{2} \in \mathfrak{R}^{3 \times 3}$, and $Q \in \mathfrak{R}^{6 \times 6}$ be positive definite. Then the control law

$$
\begin{gathered}
\dot{\hat{\alpha}}=Q^{-1}[F(\omega, C, v, \dot{v})+G(\omega, \varepsilon, \eta)]^{T}\left[\omega+K_{1} \varepsilon\right] \\
u=-[F(\omega, C, \nu, \dot{v})+G(\omega, \varepsilon, \eta)] \hat{\alpha}-\left(K_{2} K_{1}+I_{3 \times 3}\right) \varepsilon-K_{2} \omega
\end{gathered}
$$

where $G: \mathfrak{R}^{3} \times \mathfrak{R}^{3} \times \mathfrak{R} \rightarrow \mathfrak{R}^{3 \times 6}$ is defined by

$$
G(\hat{\omega}, \hat{\varepsilon}, \hat{\eta}) \triangleq-\frac{1}{2} L\left[K_{1}\left(\hat{\varepsilon}^{\times} \hat{\omega}+\hat{\eta} \hat{\omega}\right)\right]
$$


and $F$ is given by Eq. (21), solves the tracking problem. Furthermore, $\hat{\alpha}$ is bounded for all $t \geq 0$ and $\dot{\hat{\alpha}} \rightarrow 0$ as $t \rightarrow \infty$.

Proof. Define $\sigma, \gamma$, and $\beta$ by

$$
\begin{gathered}
\sigma=\omega+K_{1} \varepsilon \\
\gamma=\eta-1 \\
\beta=\alpha-\hat{\alpha}
\end{gathered}
$$

Using Eqs. (16-18), (22), and (23), we obtain the 13-dimensional system

$$
\begin{gathered}
J \dot{\sigma}=[H(\sigma, \varepsilon, \gamma, v, \dot{v})+M(\sigma, \varepsilon, \gamma)] \alpha+u \\
\dot{\varepsilon}=\frac{1}{2}\left[\varepsilon^{\times}\left(\sigma-K_{1} \varepsilon\right)+(\gamma+1)\left(\sigma-K_{1} \varepsilon\right)\right] \\
\dot{\gamma}=-\frac{1}{2} \varepsilon^{T}\left(\sigma-K_{1} \varepsilon\right) \\
Q \dot{\beta}=-[H(\sigma, \varepsilon, \gamma, v, \dot{v})+M(\sigma, \varepsilon, \gamma)]^{T} \sigma
\end{gathered}
$$

where $H: \mathfrak{R}^{3} \times \mathfrak{R}^{3} \times \mathfrak{R} \times \mathfrak{R}^{3} \times \mathfrak{R}^{3} \rightarrow \mathfrak{R}^{3 \times 6}$ is defined by

$$
H(\hat{\sigma}, \hat{\varepsilon}, \hat{\gamma}, \hat{v}, \hat{\rho}) \triangleq F\left[\hat{\sigma}-K_{1} \hat{\varepsilon}, C(\hat{\varepsilon}, \hat{\gamma}+1), \hat{v}, \hat{\rho}\right]
$$

and $M: \mathfrak{R}^{3} \times \mathfrak{R}^{3} \times \mathfrak{R} \rightarrow \mathfrak{R}^{3 \times 6}$ is defined by

$$
M(\hat{\sigma}, \hat{\varepsilon}, \hat{\gamma}) \triangleq G\left(\hat{\sigma}-K_{1} \hat{\varepsilon}, \hat{\varepsilon}, \hat{\gamma}+1\right)
$$

With this notation the control law (23) can be written as

$$
u=h(\sigma, \varepsilon, \gamma, v, \dot{v}, \beta)
$$

where $h: \Re^{3} \times \Re^{3} \times \Re \times \Re^{3} \times \Re^{3} \times \Re^{6} \rightarrow \Re^{3}$ is defined by

$$
\begin{aligned}
& h(\hat{\sigma}, \hat{\varepsilon}, \hat{\gamma}, \hat{v}, \hat{\rho}, \hat{\beta}) \triangleq-K_{2} \hat{\sigma}-\hat{\varepsilon} \\
& \quad-[H(\hat{\sigma}, \hat{\varepsilon}, \hat{\gamma}, \hat{v}, \hat{\rho})+M(\hat{\sigma}, \hat{\varepsilon}, \hat{\gamma})](\alpha-\hat{\beta})
\end{aligned}
$$

It is observed that Eqs. (28-31) are nonautonomous due to the presence of $v$ and $\dot{v}$. Under Eq. (34), we note that the origin $\left[\begin{array}{llll}\sigma^{T} & \varepsilon^{T} & \gamma & \beta^{T}\end{array}\right]=\left[\begin{array}{llll}0 & 0 & 0 & 0\end{array}\right]$ is an equilibrium solution of the system (28-31).

Next, we show that, under the control law (34), $\sigma \rightarrow 0$ and $\varepsilon \rightarrow 0$ as $t \rightarrow \infty$ for arbitrary initial conditions. To do this, consider the positive-definite candidate Lyapunov function $V: \mathfrak{R}^{3} \times \mathfrak{R}^{3} \times \mathfrak{R}^{3} \times$ $\mathfrak{R}^{6} \rightarrow \mathfrak{R}$ defined by

$$
V(\sigma, \varepsilon, \gamma, \beta)=\frac{1}{2}\left(\sigma^{T} J \sigma+\beta^{T} Q \beta\right)+\varepsilon^{T} \varepsilon+\gamma^{2}
$$

Note that $V$ is independent of time and is radially unbounded. The total time derivative of $V$ along the trajectories of the system is given by

$$
\begin{aligned}
& \dot{V}(\sigma, \varepsilon, \gamma, \beta)=-\sigma^{T} K_{2} \sigma-\varepsilon^{T} K_{1} \varepsilon \\
& \quad+\beta^{T}\left[H(\sigma, \varepsilon, \gamma, v, \dot{v})^{T} \sigma+M(\sigma, \varepsilon, \gamma)^{T} \sigma+Q \dot{\beta}\right]
\end{aligned}
$$

Using Eq. (31), we obtain the simplified expression

$$
\dot{V}(\sigma, \varepsilon, \gamma, \beta)=-\sigma^{T} K_{2} \sigma-\varepsilon^{T} K_{1} \varepsilon
$$

which shows that $\dot{V}$ is negative semidefinite and is not an explicit function of time. Because $V[\sigma(t), \varepsilon(t), \gamma(t), \beta(t)] \leq V[\sigma(0), \varepsilon(0)$, $\gamma(0), \beta(0)]$ for all $t \geq 0$ and because $V$ is radially unbounded, it follows that $\sigma, \varepsilon, \gamma$, and $\beta$ are bounded. Because by assumption $v$ and $\dot{v}$ are bounded and because $\alpha$ is constant, it follows that $H(\sigma$, $\varepsilon, \gamma, \nu, \dot{v}), M(\sigma, \varepsilon, \gamma), h(\sigma, \varepsilon, \gamma, v, \dot{v}, \beta)$, and $\hat{\alpha}$ are bounded.

Next note that the total time derivative of $\dot{V}$ along the trajectories of the system is given by

$$
\begin{aligned}
& \ddot{V}(\sigma, \varepsilon, \gamma, \beta, t)=-2 \sigma^{T} K_{2} J^{-1}\left[H(\sigma, \varepsilon, \gamma, \nu, \dot{v})^{T} \alpha\right. \\
& \left.\quad+h(\beta, \sigma, \varepsilon, \gamma, \nu, \dot{v})+M(\sigma, \varepsilon, \gamma)^{T} \alpha\right] \\
& \quad-2 \varepsilon^{T} K_{1}\left[\varepsilon^{\times} \sigma+(\gamma+1)\left(\sigma-K_{1} \varepsilon\right)\right]
\end{aligned}
$$

Because $\sigma, \varepsilon, \gamma, \beta, H(\sigma, \varepsilon, \gamma, \nu, \dot{v}), M(\sigma, \varepsilon, \gamma)$, and $h(\sigma, \varepsilon, \gamma, \nu, \dot{v}$, $\beta$ ) are bounded and $\alpha$ is constant, it follows that $\ddot{V}[\sigma(t), \varepsilon(t), \gamma(t)$, $\beta(t), t]$ is bounded for all $t \geq 0$. Using Theorem 5.4 of Ref. 10, p. 265, we conclude that $\sigma \rightarrow 0$ and $\varepsilon \rightarrow 0$. Furthermore, because $v$ and $\dot{v}$ are bounded by assumption and $\sigma \rightarrow 0$ and $\varepsilon \rightarrow 0$, it follows from Eq. (31) that $\dot{\beta} \rightarrow 0$ and thus $\dot{\hat{\alpha}} \rightarrow 0$.

Because $\sigma \rightarrow 0$ and $\varepsilon \rightarrow 0$, it follows from Eq. (25) that $\omega \rightarrow 0$. Hence we conclude that Eqs. (22) and (23) solve the tracking problem.

Remark 1. The system (28-31) has two equilibrium points, namely, $\left[\begin{array}{llll}\sigma^{T} & \varepsilon^{T} & \gamma & \beta^{T}\end{array}\right]=\left[\begin{array}{llll}0 & 0 & 0 & 0\end{array}\right]$ and $\left[\begin{array}{llll}\sigma^{T} & \varepsilon^{T} & \gamma & \beta^{T}\end{array}\right]=$ $\left[\begin{array}{llll}0 & 0 & -2 & 0\end{array}\right]$, both of which correspond to exactly the same physical orientation.

Remark 2. It can be seen from the proof of Theorem 1 that the equilibrium point $\left[\begin{array}{llll}\sigma^{T} & \varepsilon^{T} & \gamma & \beta^{T}\end{array}\right]=\left[\begin{array}{llll}0 & 0 & 0 & 0\end{array}\right]$ is uniformly stable (see Theorem 4.1 of Ref. 7) under the control law of Eqs. (22) and (23).

We observe that the control law of Eqs. (22) and (23) is global because for arbitrary initial conditions $\varepsilon \rightarrow 0$ and $\omega \rightarrow 0$ as $t \rightarrow \infty$, which ensures that tracking is achieved asymptotically. The control law given by Eq. (23) is a sixth-order proportional-integral compensator. Note that the control law requires knowledge of only $\omega$, $\varepsilon$, and $\eta$ and not of the inertia of the spacecraft.

The control law of Eqs. (22) and (23) is adaptive in the sense of Ref. 10, Chapter 1 . The state $\hat{\alpha}$ representsadjustableparameters that, under certain conditions (see Sec. IV), converge to $\alpha$. Equation (22) represents the mechanism for adjusting these parameters. The state $\hat{\alpha}$ is consequently termed the adaptive parameter. Although the time derivative of the adaptive parameter converges to zero as $t \rightarrow \infty$, $\hat{\alpha}$ does not necessarily converge. It is mathematically possible for the derivative of a differentiable function to converge to zero, and yet the function need not converge. For example, consider $f(x)=$ $\cos [\log (1+x)]$ for $x \rightarrow \infty$. Then $\lim _{x \rightarrow \infty} f^{\prime}(x)=0$, whereas $\lim _{x \rightarrow \infty} f(x)$ does not exist.

The control law of Eqs. (22) and (23) can be written as

$$
\begin{aligned}
\dot{\hat{\alpha}} & =-Q^{-1}\left[L(C \dot{v})+(C v)^{\times} L(C v)\right]^{T}\left(\omega+K_{1} \varepsilon\right) \\
& -Q^{-1}\left\{-L\left(\omega^{\times} C v\right)+\omega^{\times} L(\omega+C v)+(C v)^{\times} L(\omega)\right. \\
& \left.+\frac{1}{2} L\left[K_{1}\left(\varepsilon^{\times} \omega+\eta \omega\right)\right]\right\}^{T}\left(\omega+K_{1} \varepsilon\right) \\
u & =-K_{2} K_{1} \varepsilon-K_{2} \omega+\left[L(C \dot{v})+(C v)^{\times} L(C v)\right] \hat{\alpha} \\
& -\varepsilon+\left\{-L\left(\omega^{\times} C v\right)+\omega^{\times} L(\omega+C v)+(C v)^{\times} L(\omega)\right. \\
& \left.+\frac{1}{2} L\left[K_{1}\left(\varepsilon^{\times} \omega+\eta \omega\right)\right]\right\} \hat{\alpha}
\end{aligned}
$$

Replacing $K_{1}$ by $k_{1} I_{3 \times 3}$ in Eq. (38), $K_{2}$ by $k_{2} I_{3 \times 3}$, and $K_{1}$ by $k_{3} I_{3 \times 3}$ in Eq. (39), where $k_{1}>0, k_{2}>0$, and $k_{3}>0$, and omitting terms in Eqs. (38) and (39), we obtain the simplified control law

$$
\begin{aligned}
& \dot{\hat{\alpha}}=-Q^{-1}\left[L(C \dot{v})+(C v)^{\times} L(C v)\right]^{T}\left(\omega+k_{1} \varepsilon\right) \\
& u=-k_{2} k_{3} \varepsilon-k_{2} \omega+\left[L(C \dot{v})+(C v)^{\times} L(C v)\right] \hat{\alpha}
\end{aligned}
$$

Now let

$$
0<k_{1}<\min \left\{\frac{4 k_{2} k_{3}^{2}}{4 \lambda_{1} k_{2} k_{3}+9 \mu^{2} \lambda_{1}^{2}}, \frac{k_{2} \lambda_{3}+\sqrt{k_{2}^{2} \lambda_{3}^{2}+4 k_{2} k_{3} \lambda_{3}^{2} \lambda_{1}^{2}}}{2 \lambda_{1}^{2}}\right\}
$$

where

$$
\mu=\sup _{t \geq 0} \sqrt{v(t)^{T} v(t)}
$$

and where $\lambda_{1}$ is the largest eigenvalue of $J$ and $\lambda_{3}$ is the smallest eigenvalue of $J$. Then by using the Lyapunov function

$$
V=k_{2}\left(k_{1}+k_{3}\right)\left[(\eta-1)^{2}+\varepsilon^{T} \varepsilon\right]+\frac{1}{2} \omega^{T} J \omega+k_{1} \varepsilon^{T} J \omega
$$

it is shown in Ref. 8 that tracking is achieved for this simplified adaptive controllaw. Knowledge of the largest and smallest principal 
moments of inertia is required to guarantee that tracking is achieved by the control law of Eqs. (40) and (41).

We now consider the convergence of $\hat{\alpha}$ for periodic command signals.

Theorem 2. Assume that $v$ is periodic, define $W:[0, \infty) \rightarrow$ $\mathfrak{R}^{3 \times 6}$ by

$$
W(t) \triangleq L[\dot{v}(t)]+v(t)^{\times} L[v(t)]
$$

and let

$$
G=\{\chi: W(t) \chi=0 \text { for all } t \geq 0\}
$$

Under the control law given by Eqs. (22) and (23), $\alpha-\hat{\alpha} \rightarrow G$ as $t \rightarrow \infty$.

Proof. With $\sigma, \gamma$, and $\beta$ defined by Eqs. (25-27), respectively, we obtain the differential equations (28-31), where $H$ and $M$ are defined by Eqs. (32) and (33), respectively, and $u=h(\sigma, \varepsilon, \gamma, v, \dot{v}$, $\beta$ ), where $h$ is defined by Eq. (35). Note that because $v$ is periodic and differentiable, $\dot{v}$ is periodic.

Consider the candidate Lyapunov function $V$ defined by Eq. (36). Now $V$ is $C^{1}$, positive definite, and radially unbounded, and $V$ along the trajectories of the system is given by Eq. (37).

Let $x=\left[\begin{array}{llll}\sigma^{T} & \varepsilon^{T} & \gamma & \beta^{T}\end{array}\right]^{T}$ and let $E_{0}=\{x: \dot{V}(x)=0\}$. Let $x\left(t ; x_{0}, t_{0}\right)$ denote the solution of the system (28-31) at time $t \geq t_{0}$ where $x\left(t_{0} ; x_{0}, t_{0}\right)=x_{0}$. Let $L=\left\{(\hat{x}, \hat{t}) \in E_{0} \times[0, \infty): x(t ; \hat{x}\right.$, $\hat{t}) \in E_{0}$ for all $\left.t \geq \hat{t}\right\}$ and $N=\{x(t ; \hat{x}, \hat{t}):(\hat{x}, \hat{t}) \in L, t \geq \hat{t}\}$. Note that $N \subset E_{0}$. Using Eqs. (8), (26), and (28-31), it follows that $N$ is given by

$$
N=\{(\sigma, \varepsilon, \gamma, \beta): \sigma=0, \varepsilon=0, \gamma \in\{-2,0\}, \beta \in G\}
$$

It now follows from Theorem 2.8 of Ref. 15 that $\alpha-\hat{\alpha} \rightarrow G$.

The following corollary of Theorem 2 considers the special case in which $v$ is constant. Define

$$
G_{0}=\left\{\chi: v^{\times} L(v) \chi=0\right\}
$$

Corollary 1. Assume that $v$ is constant. Under the control law given by Eqs. (22) and (23), $\alpha-\hat{\alpha} \rightarrow G_{0}$, where $G_{0}$ is given by Eq. (44) as $t \rightarrow \infty$. $G_{0}$.

Proof. Because $v$ is constant, Theorem 2 implies that $\alpha-\hat{\alpha} \rightarrow$

We now consider the case in which $v$ represents a constant spin about one of the principal axes that is equivalent to $v^{\times} L(v) \alpha=0$. For such a command, it is desirable that the control law satisfy $u \rightarrow 0$ as $t \rightarrow \infty$. The following result shows that the control law of Eqs. (22) and (23) has this property.

Corollary2. Assume that $v$ is constantand satisfies $v^{\times} L(v) \alpha=0$. Then, under the control law given by Eqs. (22) and (23), $u \rightarrow 0$ as $t \rightarrow \infty$.

Proof. Under the control law given by Eqs. (22) and (23), $\omega \rightarrow 0$ and $\varepsilon \rightarrow 0$, which implies that $u+v^{\times} L(v) \hat{\alpha} \rightarrow 0$ as $t \rightarrow \infty$. Because $v^{\times} L(v) \alpha=0$, it follows from Corollary 1 that $\hat{\alpha} \rightarrow G_{0}$, where $G_{0}$ is given by Eq. (44). It thus follows that $u \rightarrow 0$ as $t \rightarrow$ $\infty$.

\section{Inertia Matrix Identification}

In this section, we present a method for identifying the spacecraft inertia matrix. We first use Corollary 1 to identify the off-diagonal terms $J_{12}, J_{23}$, and $J_{31}$.

Proposition 1. Let $v$ be constant. If $v=\left[\begin{array}{lll}0 & v_{2} & 0\end{array}\right]^{T}$, where $v_{2} \neq 0$, then, under the control law given by Eqs. (22) and (23), $\hat{\alpha}_{4} \rightarrow \alpha_{4}$ and $\hat{\alpha}_{6} \rightarrow \alpha_{6}$ as $t \rightarrow \infty$. Furthermore, if $v=\left[\begin{array}{lll}0 & 0 & v_{3}\end{array}\right]^{T}$, where $v_{3} \neq 0$, then, under the control law given by Eqs. (22) and (23), $\hat{\alpha}_{4} \rightarrow \alpha_{4}$ and $\hat{\alpha}_{5} \rightarrow \alpha_{5}$ as $t \rightarrow \infty$.

Proof. From Corollary 1, under the control law given by Eqs. (22) and (23), $\alpha-\hat{\alpha} \rightarrow G_{0}$, where $G_{0}$ is given by Eq. (44). Now $v^{\times} L(v)$ is computed to be

$$
v^{\times} L(v)=\left[\begin{array}{cccccc}
0 & 0 & 0 & v_{2}^{2} & 0 & 0 \\
0 & 0 & 0 & 0 & 0 & 0 \\
0 & 0 & 0 & 0 & 0 & -v_{2}^{2}
\end{array}\right]
$$

It thus follows that $v^{\times} L(v) \chi=0$ if and only if $\chi_{4}=0$ and $\chi_{6}=0$ where $\chi=\left[\begin{array}{llllll}\chi_{1} & \chi_{2} & \chi_{3} & \chi_{4} & \chi_{5} & \chi_{6}\end{array}\right]^{T}$. Thus $\hat{\alpha}_{4} \rightarrow \alpha_{4}$ and $\hat{\alpha}_{6} \rightarrow \alpha_{6}$ as $t \rightarrow \infty$. If $v=\left[\begin{array}{lll}0 & 0 & v_{3}\end{array}\right]^{T}$, where $v_{3} \neq 0$, then $v^{\times} L(v)$ is computed to be

$$
v^{\times} L(v)=\left[\begin{array}{cccccc}
0 & 0 & 0 & -v_{3}^{2} & 0 & 0 \\
0 & 0 & 0 & 0 & v_{3}^{2} & 0 \\
0 & 0 & 0 & 0 & 0 & 0
\end{array}\right]
$$

It now follows that $\hat{\alpha}_{4} \rightarrow \alpha_{4}$ and $\hat{\alpha}_{5} \rightarrow \alpha_{5}$ as $t \rightarrow \infty$.

Hence the off-diagonal terms $J_{12}, J_{13}$, and $J_{23}$ can be identified by performing two constant tracking maneuvers. We now consider periodic maneuvers for identifying the entire inertia matrix.

Proposition 2. Let $v$ be periodic and let $W(t)$ be given by Eq. (42). Furthermore, let $0 \leq t_{1} \leq t_{2} \leq \cdots \leq t_{n}$ and suppose that

$$
\operatorname{rank}\left[\begin{array}{c}
W\left(t_{1}\right) \\
\vdots \\
W\left(t_{n}\right)
\end{array}\right]=6
$$

Then, under the control law given by Eqs. (22) and (23), $\hat{\alpha} \rightarrow \alpha$.

Proof. From Theorem 2 it follows that $\alpha-\hat{\alpha} \rightarrow G$, where $G$ is given by Eq. (24). However, it follows from Eq. (45) that $G=\{0\}$. Hence $\hat{\alpha} \rightarrow \alpha$.

There are many signals that satisfy the conditions of Proposition 2. Consider, for example, the periodic signal

$$
v(t)=\left[\begin{array}{lll}
\sin t & \sin 2 t & \sin 3 t
\end{array}\right]^{T}
$$

Then, with $t_{1}=0$ and $t_{2}=\pi / 2$, we obtain

$$
\left[\begin{array}{c}
W(0) \\
W(\pi / 2)
\end{array}\right]=\left[\begin{array}{rrrrrr}
1 & 0 & 0 & 0 & 3 & 2 \\
0 & 2 & 0 & 3 & 0 & 1 \\
0 & 0 & 3 & 2 & 1 & 0 \\
0 & 0 & 0 & -1 & 0 & -1 \\
-1 & -2 & 1 & 0 & 0 & 0 \\
0 & 0 & 0 & -3 & 0 & 1
\end{array}\right]
$$

The maximum and minimum singular values of

$$
\left[\begin{array}{c}
W(0) \\
W(\pi / 2)
\end{array}\right]
$$

are 5.2406 and 0.3512 , respectively. Therefore

$$
\left[\begin{array}{c}
W(0) \\
W(\pi / 2)
\end{array}\right]
$$

is well conditioned and

$$
\operatorname{rank}\left[\begin{array}{c}
W(0) \\
W(\pi / 2)
\end{array}\right]=6
$$

Hence, under the control law given by Eqs. (22) and (23), $\hat{\alpha} \rightarrow \alpha$. Thus the inertia matrix can be identified using a single periodic command signal.

\section{Numerical Simulations}

In this section, we present simulations to illustrate tracking and identification of the spacecraft inertia matrix. The two tracking maneuvers considered are a constant spin about an axis fixed to the spacecraft and a coning motion. We choose the axis fixed to the spacecraft to be coincident with the intermediate principal axis. The maneuver given by Eq. (46) is used to identify the spacecraftinertia The same initial conditions are chosen for the three maneuvers. The initial angular velocity is $\Omega=\left[\begin{array}{lll}0.4 & 0.2 & -0.1\end{array}\right]^{T} \mathrm{rad} / \mathrm{s}$, the initial 
orientation of the spacecraft is given by $\epsilon=\left[\begin{array}{lll}-0.1 & 0.1 & -0.1\end{array}\right]^{T}$, $\zeta=0.9849$, and the initial value of the adaptive parameter $\hat{\alpha}$ in kilogram-square meter is $\hat{\alpha}=\left[\begin{array}{llllll}22 & 18 & 13 & 1.6 & 1.0 & 1.3\end{array}\right]^{T}$.

First we command an axis fixed in the spacecraft, namely, the $Y_{\mathcal{B}_{\mathrm{cm}}}$ axis of $\mathcal{B}_{\mathrm{cm}}$, to point in a given inertial direction while the spacecraft performs a constant spin about this axis. We perform the simulation for the case in which $Y_{\mathcal{B}_{\mathrm{cm}}}$ coincides with the intermediate principal axis. We assume no knowledge of the moments of inertia of the spacecraft. The maneuver is performed using the control law given by Theorem 1 . In the body frame $\mathcal{B}_{\mathrm{cm}}$, let

$$
J=\left[\begin{array}{ccc}
20 & 0 & 0.9 \\
0 & 17 & 0 \\
0.9 & 0 & 15
\end{array}\right]
$$

so that $Y_{\mathcal{B}_{\mathrm{cm}}}$ is the intermediateprincipalaxis; let $\nu=\left[\begin{array}{lll}0 & 1 & 0\end{array}\right]^{T} \mathrm{rad} / \mathrm{s}$; let the initial orientation be $\xi=\left[\begin{array}{lll}0 & 0 & 0\end{array}\right]^{T}$ and $\mu=1$; and let $X, Y$, and $Z$ be the axes of the inertial frame. Then the desired motion is a spin about the intermediate principal axis, with the intermediate principal axis aligned with the $Y$ axis of the inertial frame. The gains are chosen to be $K_{1}=20 I_{3 \times 3}, K_{2}=5 I_{3 \times 3}$, and $Q=I_{6 \times 6}$.

Applying the control law given by Theorem 1, we observe from Figs. 1 and 2 that tracking is achieved. Figures 3 and 4 indicate that

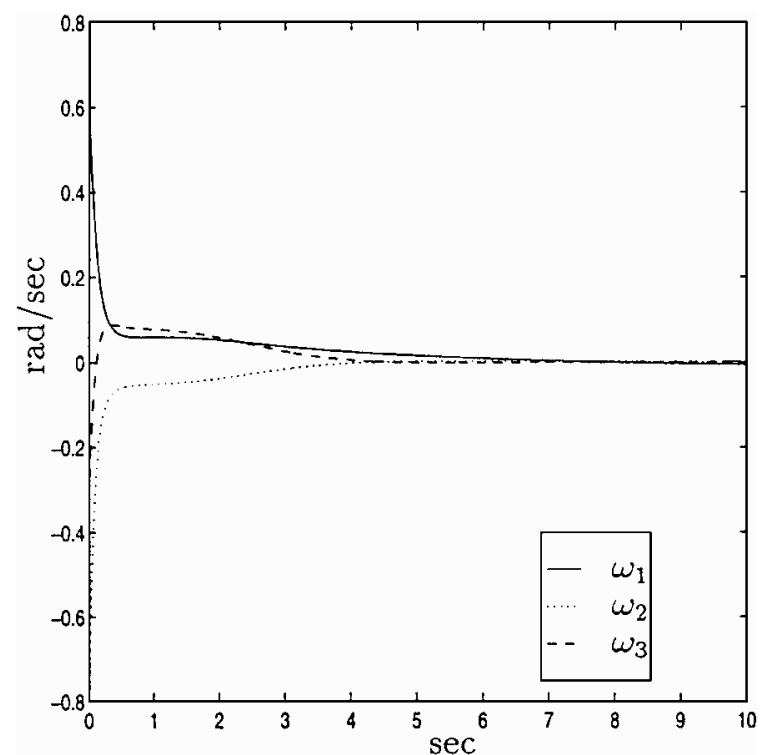

Fig. 1 Relative angular velocity $\omega=\left[\begin{array}{lll}\omega_{1} & \omega_{2} & \omega_{3}\end{array}\right]^{T}$.

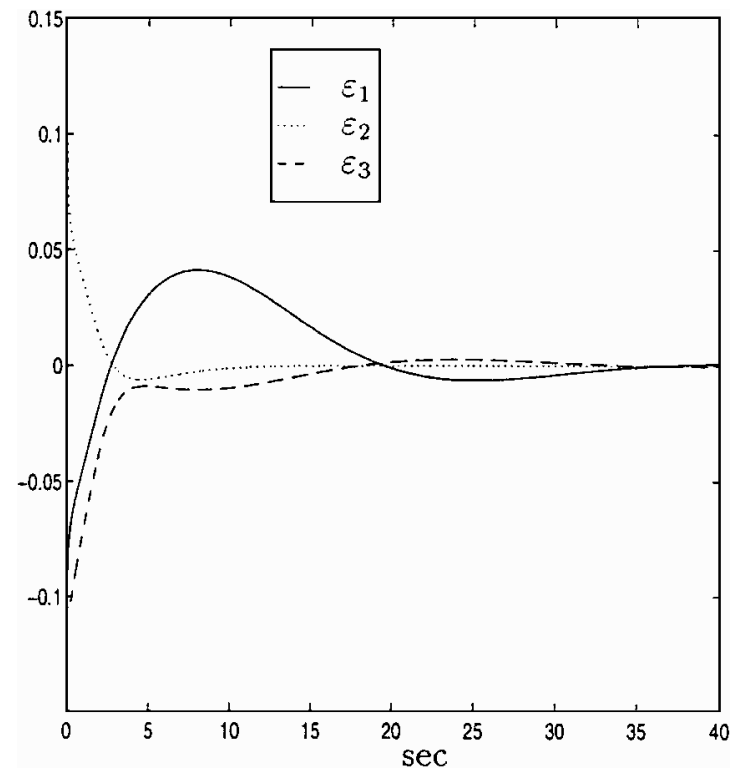

Fig. 2 Relative Euler parameter $\varepsilon=\left[\begin{array}{lll}\varepsilon_{1} & \varepsilon_{2} & \varepsilon_{3}\end{array}\right]^{T}$.

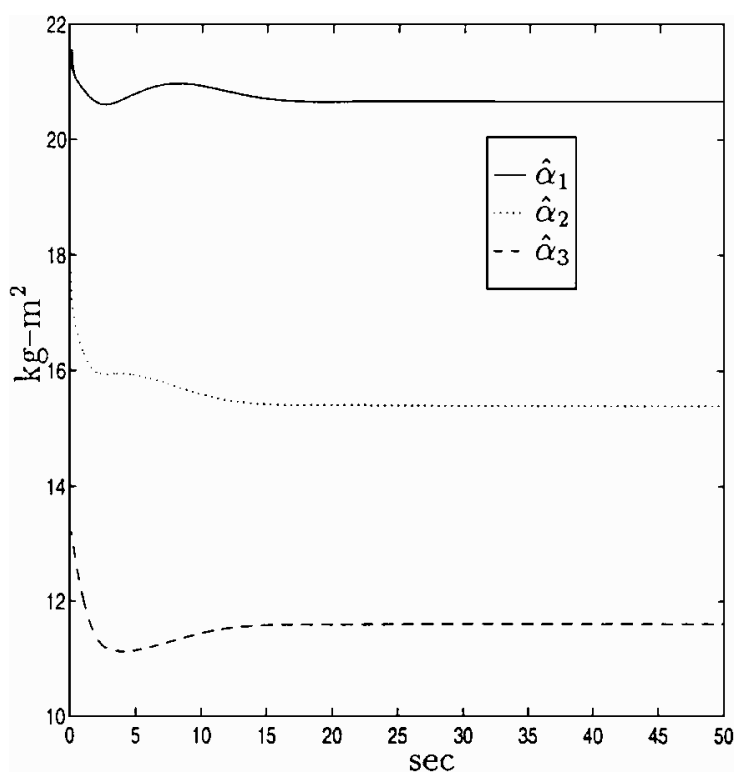

Fig. 3 Adaptive parameters $\hat{\alpha}_{1}, \hat{\alpha}_{2}$, and $\hat{\alpha}_{3}$.

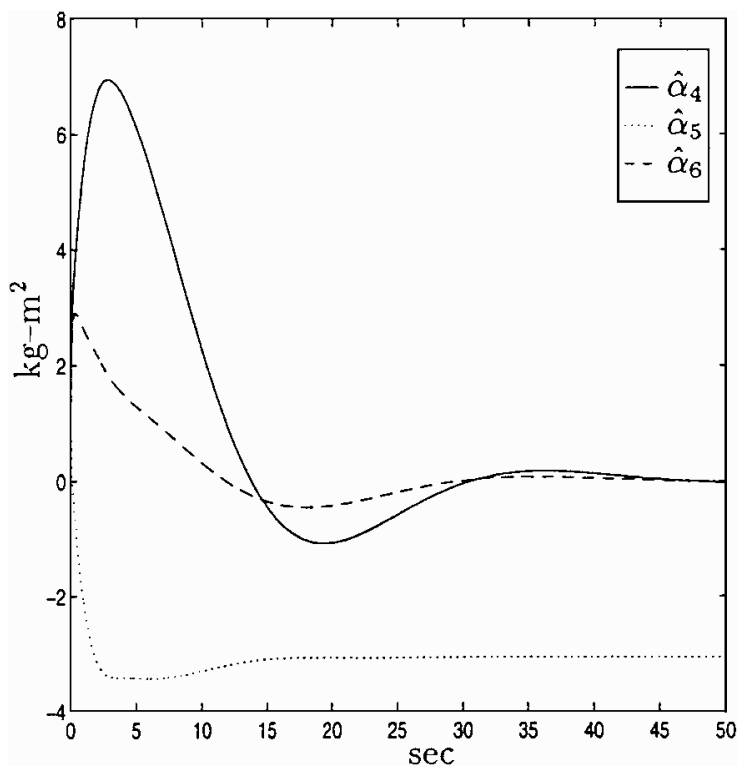

Fig. 4 Adaptive parameters $\hat{\alpha}_{4}, \hat{\alpha}_{5}$, and $\hat{\alpha}_{6}$.

although $J_{12}$ and $J_{23}$ are identified in accordance with Proposition 1, the remaining entries of the inertiamatrix are not. Figure 5 shows that $\Omega_{2}$ converges to $1 \mathrm{rad} / \mathrm{s}$, whereas $\Omega_{1}$ and $\Omega_{3}$ convergeto $0 \mathrm{rad} / \mathrm{s}$. Thus the spacecraft approaches a simple spin about its intermediate axis.

Next we command the spacecraft to perform a specified coning motion. We use 3, 2, 1 Euler angles (Ref. 16, p. 354) $\psi, \theta$, and $\phi$ to represent the precession motion of the desired frame $\mathcal{D}$. Let $\psi(t)=0.1 t \mathrm{rad}, \theta(t)=-0.2222 \pi \mathrm{rad}$, and $\phi(t)=0.5 t \mathrm{rad}$. Then the desired motion is a coning maneuver with a precession rate of $0.1 \mathrm{rad} / \mathrm{s}$, a spin rate of $0.5 \mathrm{rad} / \mathrm{s}$, and a coning angle of $40 \mathrm{deg}$. The angular velocity $v$ and the initial values of the Euler parameters $\xi$ and $\mu$ are computed from Ref. 14 using Eq. (24), page 27, Table 2.1, page 20, and Eqs. (15) and (16), page 18. The inertia matrix $J$ of the spacecraft in kilogram-square meter is given by

$$
J=\left[\begin{array}{ccc}
20 & 1.2 & 0.9 \\
1.2 & 17 & 1.4 \\
0.9 & 1.4 & 15
\end{array}\right]
$$

and the gains $K_{1}, K_{2}$, and $Q$ are chosen to be $2.5 I_{3 \times 3}, 0.7 I_{3 \times 3}$, and $I_{6 \times 6}$, respectively. The controllergiven by Theorem 1 is used, and it is observed from Figs. 6 and 7 that $\omega \rightarrow 0$ and $\epsilon \rightarrow 0$. However, note from Figs. 8 and 9 that $\hat{\alpha}$ does not converge to $\alpha$. Figure 10 shows 


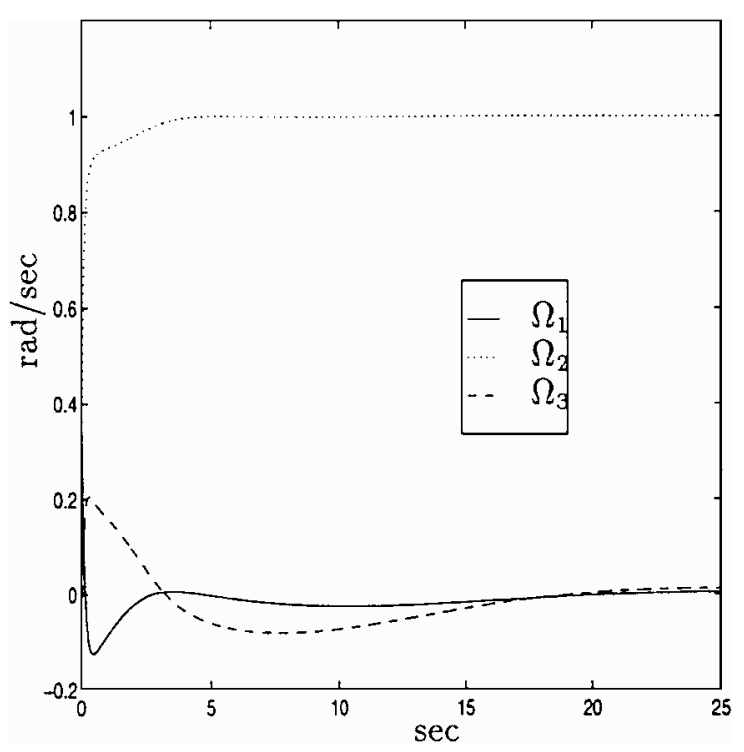

Fig.5 Angular velocity $\Omega=\left[\begin{array}{lll}\Omega_{1} & \Omega_{2} & \Omega_{3}\end{array}\right]^{T}$ of $\mathcal{B}$ with respect to inertial.

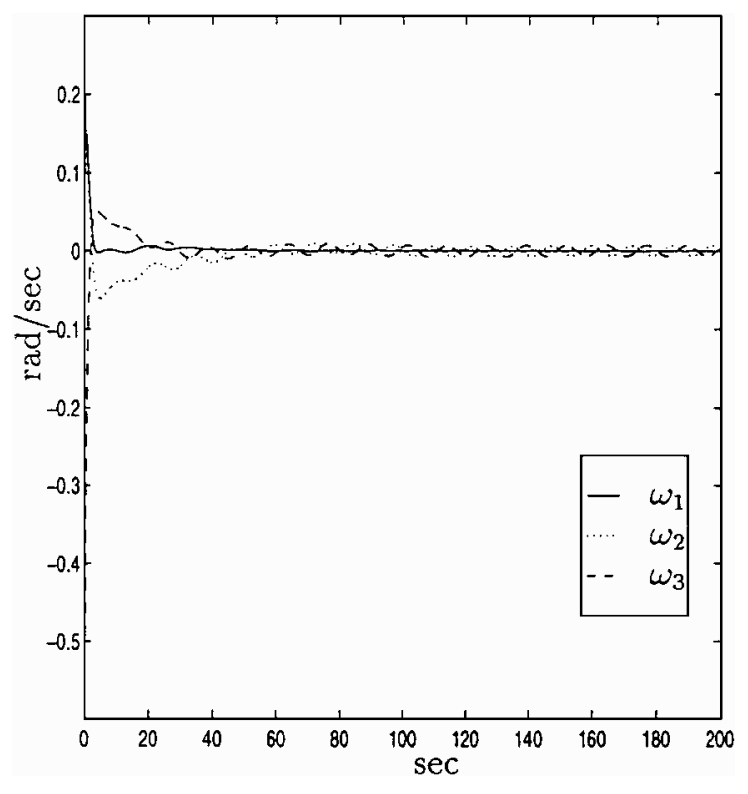

Fig. 6 Relative angular velocity $\omega=\left[\begin{array}{lll}\omega_{1} & \omega_{2} & \omega_{3}\end{array}\right]^{T}$.

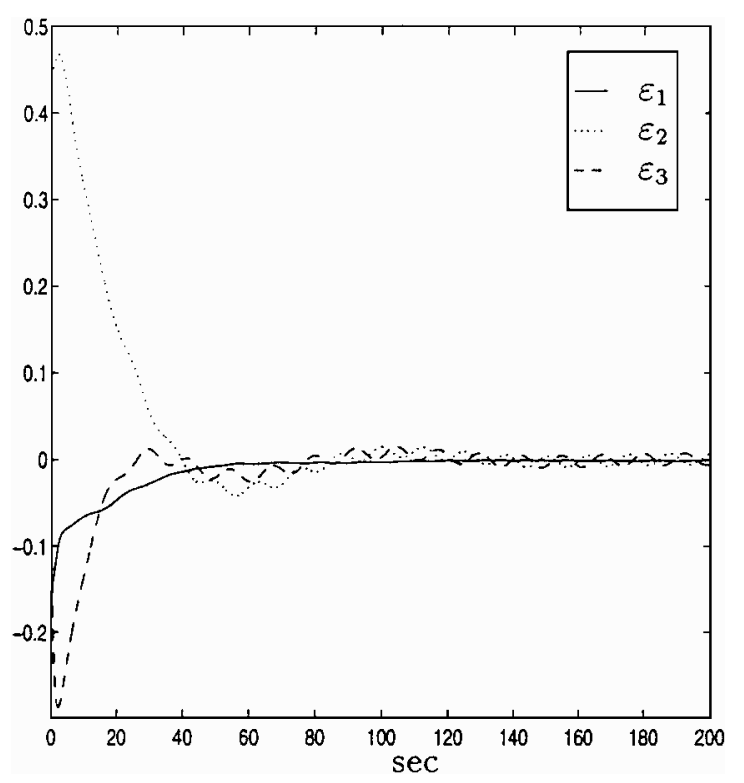

Fig. 7 Relative Euler parameter $\varepsilon=\left[\begin{array}{lll}\varepsilon_{1} & \varepsilon_{2} & \varepsilon_{3}\end{array}\right]^{T}$.

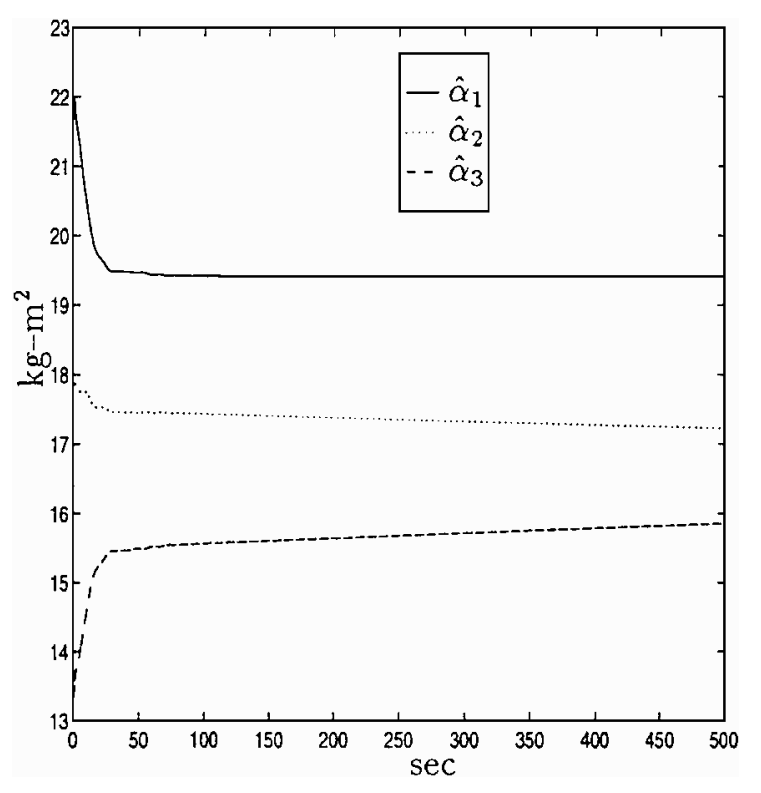

Fig. 8 Adaptive parameters $\hat{\alpha}_{1}, \hat{\alpha}_{2}$, and $\hat{\alpha}_{3}$.

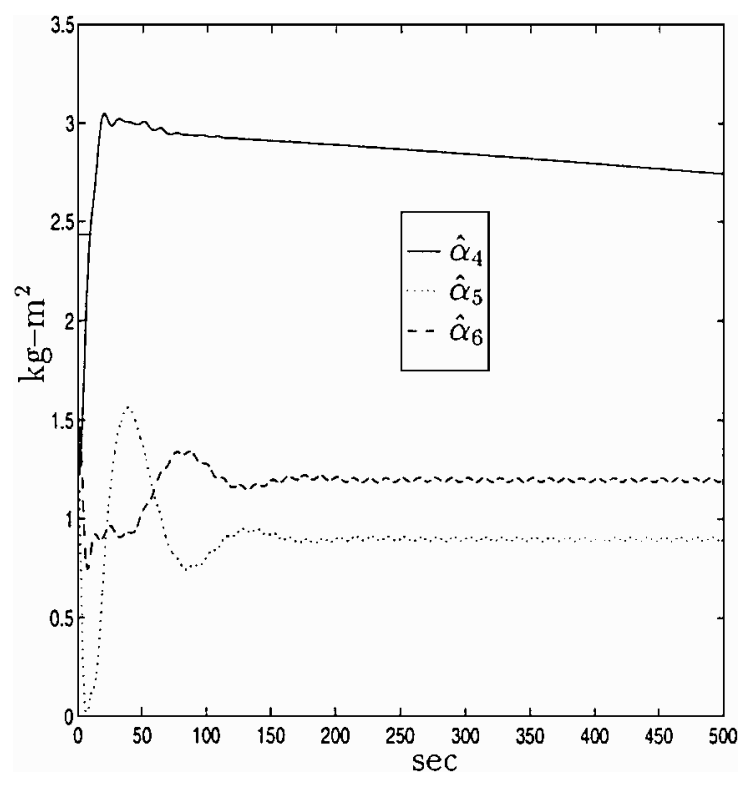

Fig. 9 Adaptive parameters $\hat{\alpha}_{4}, \hat{\alpha}_{5}$, and $\hat{\alpha}_{6}$.

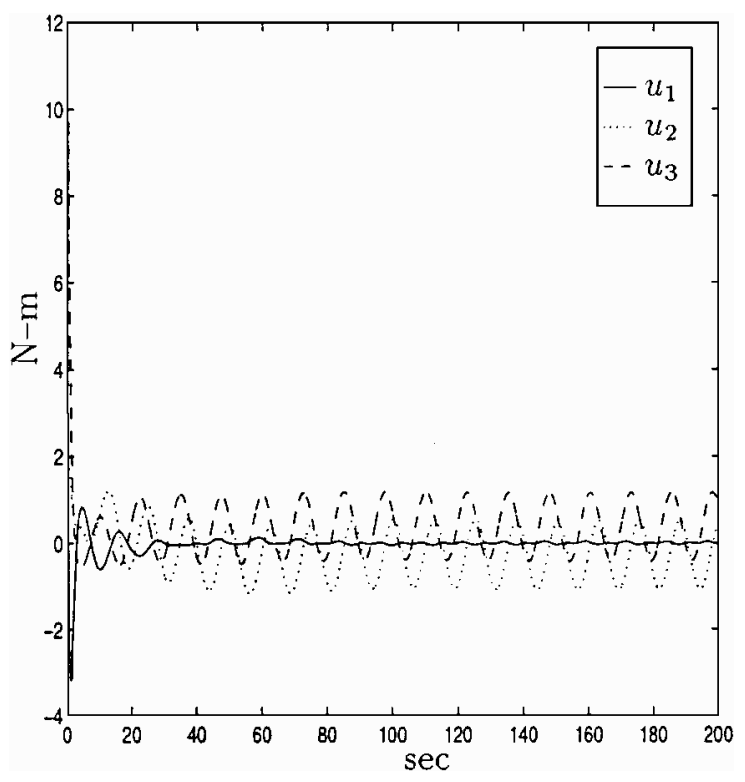

Fig. 10 Control torque $u=\left[\begin{array}{lll}u_{1} & u_{2} & u_{3}\end{array}\right]^{T}$. 


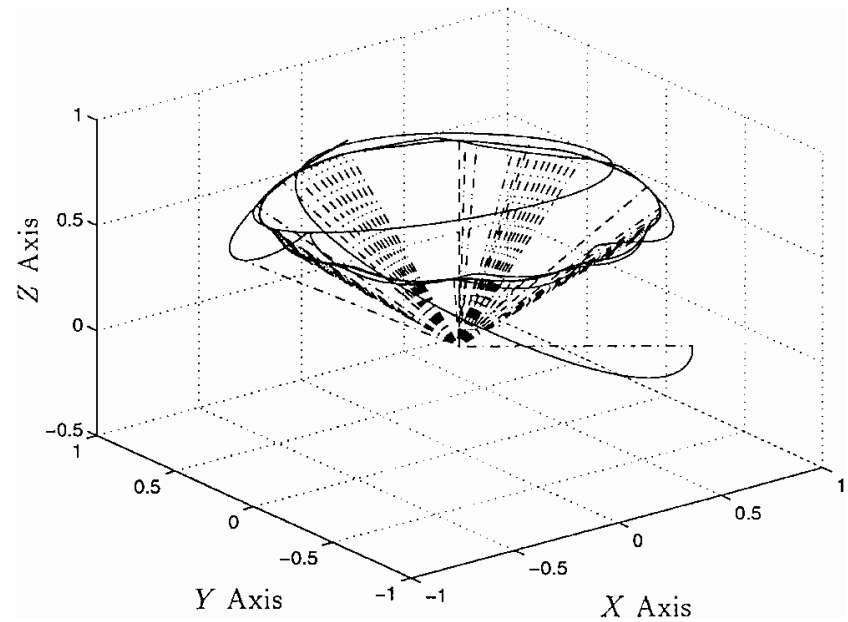

Fig. 11 Coning motion.

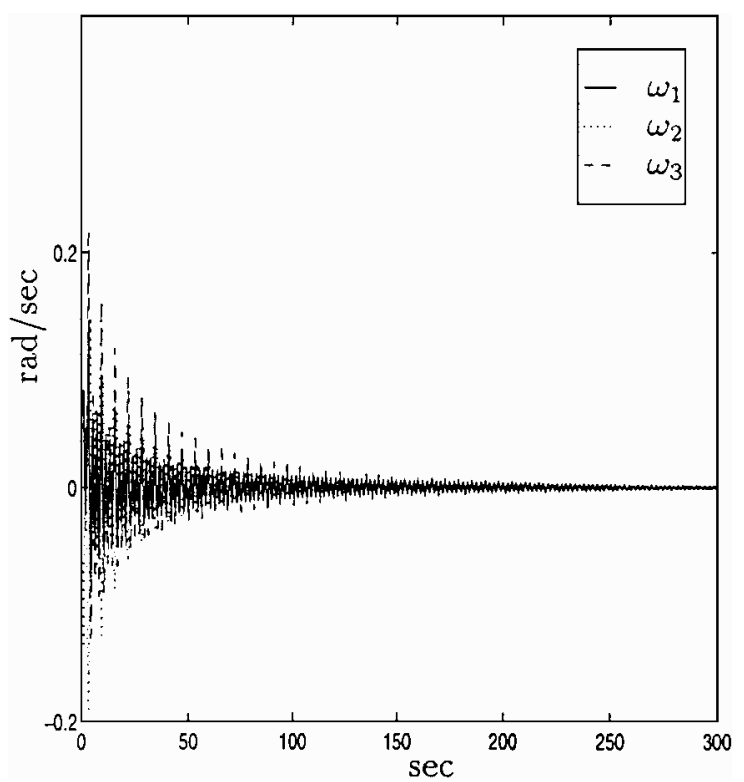

Fig. 12 Relative angular velocity $\omega=\left[\begin{array}{lll}\omega_{1} & \omega_{2} & \omega_{3}\end{array}\right]^{T}$.

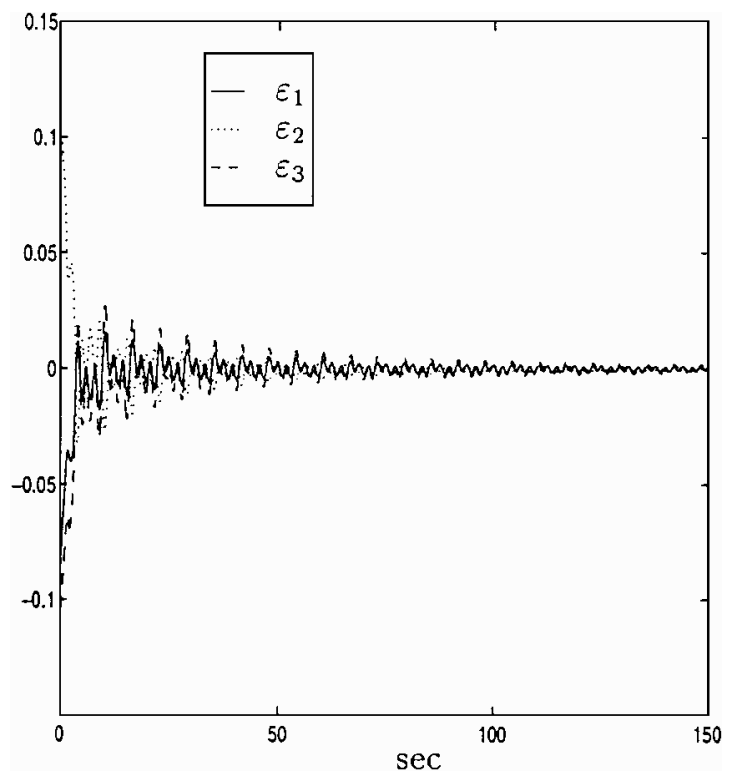

Fig. 13 Relative Euler parameter $\varepsilon=\left[\begin{array}{lll}\varepsilon_{1} & \varepsilon_{2} & \varepsilon_{3}\end{array}\right]^{T}$.

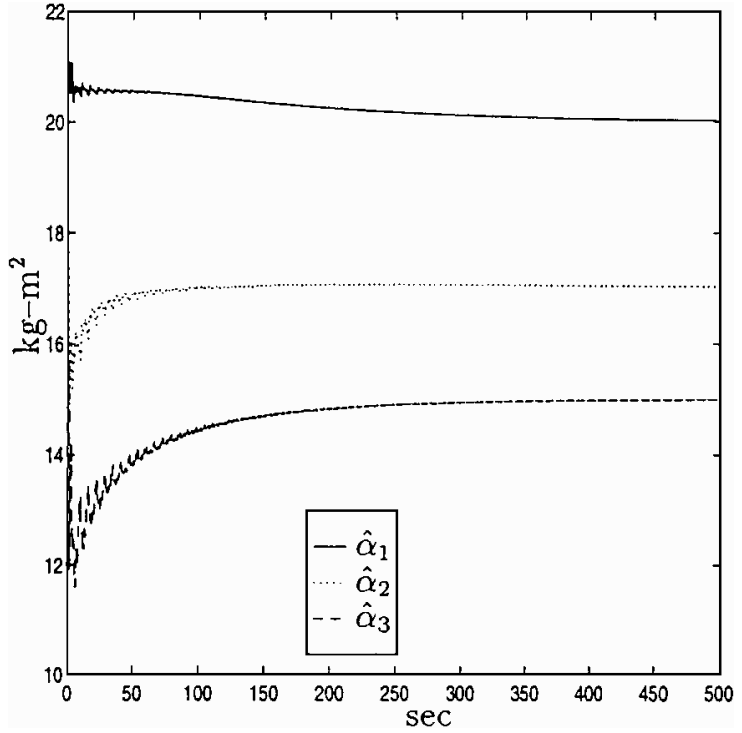

Fig. 14 Adaptive parameters $\hat{\alpha}_{1}, \hat{\alpha}_{2}$, and $\hat{\alpha}_{3}$.

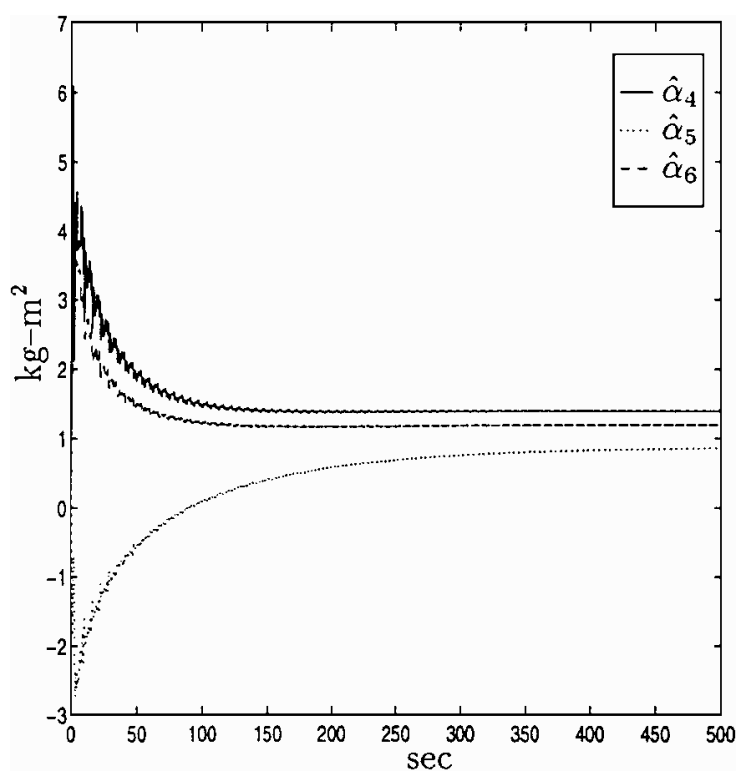

Fig. 15 Adaptive parameters $\hat{\alpha}_{4}, \hat{\alpha}_{5}$, and $\hat{\alpha}_{6}$.

the control history. It is observed from numerical simulations that decreasing the gains tends to decrease the maximum control torque and to increase the settling time. Figure 11 shows the motion of the $X_{\mathcal{B}_{\mathrm{cm}}}$ axis of the spacecraft for a period of $500 \mathrm{~s}$. The plots indicate that the desired coning motion is achieved.

To identify the inertia matrix, the maneuver $v(t)=$ $\left[\begin{array}{lll}\sin t & \sin 2 t & \sin 3 t\end{array}\right]^{T} \mathrm{rad} / \mathrm{s}$ described in Sec. IV is chosen. The initial orientation of $\mathcal{D}$ is chosen to be $\xi=\left[\begin{array}{lll}0 & 0 & 0\end{array}\right]^{T}$ and $\mu=1$, and the inertia matrix is given by Eq. (47). The gains are chosen to be $K_{1}=20 I_{3 \times 3}, K_{2}=5 I_{3 \times 3}$, and $Q=I_{6 \times 6}$. Under the control law given by Eqs. (22) and (23), we observe from Figs. 12 and 13 that $\omega \rightarrow 0$ and $\varepsilon \rightarrow 0$. Furthermore, Figs. 14 and 15 indicate that $\hat{\alpha} \rightarrow \alpha$ in accordance with Proposition 2. It is noted from the numerical simulations that tracking is achieved rapidly, whereas parameter identification takes much longer.

\section{Conclusions}

An adaptive feedback control algorithm has been developed to provide global tracking of commanded spacecraft motion. The algorithm assumes no knowledge of the inertia of the spacecraft and is thus unconditionally robust with respect to this parametric uncertainty. It was shown using a Lyapunov argument that the attitude and 
angular velocity tracking error converge to zero. Furthermore, the control algorithm was used to identify the spacecraft inertia matrix. Numerical simulations illustrate tracking and identification of the inertia matrix.

\section{Acknowledgments}

This research was supported in part by the U.S. Air Force Office of Scientific Research under Grant F49620-95-1-0019 and the University of Michigan's Office of the Vice President for Research.

\section{References}

${ }^{1}$ Junkins, J. L., and Turner, J. D., "Optimal Continuous Torque Attitude Maneuvers," Journal of Guidance and Control, Vol. 3, No. 3, 1980, pp. 210-217.

${ }^{2}$ Skaar, S. B., and Kraige, L. G., "Large Angle Spacecraft Attitude Maneuvers Using an Optimal Reaction Wheel Power Criterion," Journal of Astronautical Sciences, Vol. 32, No. 1, 1984, pp. 47-61.

${ }^{3}$ Wie, B., Weiss, H., and Arapostathis, A., "Quaternion Feedback Regulator for Spacecraft Eigenaxis Rotations," Journal of Guidance, Control, and Dynamics, Vol. 8, No. 3, 1989, pp. 360-365.

${ }^{4}$ Joshi, S. M., Kelkar, A. G., and Wen, J. T.-Y., "Robust Attitude Stabilization of Spacecraft Using Nonlinear Quaternion Feedback," IEEE Transactions on Automatic Control, Vol. 40, Oct. 1995, pp. 1800-1803.

${ }^{5}$ Singh, S. N., "Nonlinear Adaptive Attitude Control of Spacecraft," IEEE Transactions on Aerospace Electrical Systems, Vol. AES-23, No. 3, 1987.
${ }^{6}$ Sheen, J. J., and Bishop, R. H., "Adaptive Nonlinear Control of Spacecraft," Proceedings of the American Control Conference, 1994, pp. 2867 2871.

${ }^{7}$ Slotine, J. J. E., and Li, W., Applied Nonlinear Control, PrenticeHall, Englewood Cliffs, NJ, 1989.

${ }^{8}$ Wen, J. T., and Kreutz-Delgado, K., "The Attitude Control Problem," IEEE Transactions on Automatic Control, Vol. 36, No. 10, 1991, pp. 11481162.

${ }^{9}$ Zaremba, A. T., "An Adaptive Scheme with Parameter Identification for Spacecraft Attitude Control," Proceedings of the American Control Conference, 1997, pp. 552-556.

${ }^{10}$ Astrom, K. J., and Wittenmark, B., Adaptive Control, 2nd ed., AddisonWesley, Reading, MA, 1995.

${ }^{11}$ Hsu, P., Bodson, M., Sastry, S., and Paden, B., "Adaptive Identification and Control for Manipulators Without Using Joint Accelerations," Proceedings of the International Conference on Robotics and Automation, 1987, pp. $1210-1215$.

${ }^{12}$ Bloch, A. M., and Marsden, J. E., "Stabilization of Rigid Body Dynamics by the Energy-Casimir Method," Systems and Control Letters, 14, 1990, pp. 341-346.

${ }^{13}$ Aeyels, D., "On Stabilization by Means of the Energy-Casimir Method," Systems and Control Letters, 18, 1992, pp. 325-328.

${ }^{14}$ Hughes, P. C., Spacecraft Attitude Dynamics, Wiley, New York, 1986.

${ }^{15}$ Narendra, K. S., and Annaswamy, A. M., Stable Adaptive Systems, Prentice-Hall, Englewood Cliffs, NJ, 1989.

${ }^{16}$ Greenwood, D. T., Principles of Dynamics, Prentice-Hall, Englewood Cliffs, NJ, 1988. 